GANIT J. Bangladesh Math. Soc. (ISSN 1606-3694) 34 (2014) 101-110

\title{
NUMERICAL SIMULATION OF A SECOND ORDER TRAFFIC FLOW MODEL
}

\author{
Tauhedul Azam ${ }^{1}$, M. K. Pandit ${ }^{2}$ and L. S. Andallah ${ }^{3}$ \\ Department of Mathematics, Jahangirnagar University, Savar, Dhaka, Bangladesh \\ ${ }^{1}$ Email: tauhed.ju@ gmail.com; ${ }^{2}$ Email: mkpamaths@yahoo.com, ${ }^{3}$ Email: andallahls@ gmail.com
}

Received 15.10.2014 Accepted 11.01.2015

\begin{abstract}
A second order fluid dynamical traffic flow model is considered which is a parabolic type second order partial differential equation appended with initial and boundary conditions reads as an initial boundary value problem (IBVP). For a linear velocity-density relation the paper presents the analytical solution of the model by using Cole-Hopf transformation. For the numerical solution of the IBVP, we investigate explicit central difference scheme and implement the numerical scheme by developing computer programming code. Error estimation is produced which shows the numerical solution is accurate up to eight decimal places and this result is much better than the work presented in [6]. The numerical feature of rate of convergence observed by graphical presentation is also found better than the previous work. The computed numerical simulation results are seen in a good qualitative agreement with the well known traffic flow behavior for various parameters.
\end{abstract}

Keywords: Fluid dynamical traffic flow model, Finite difference method, Numerical Simulation

\section{Introduction}

The fast growing number of vehicles on networks of roads, either highways or urban streets, and the related economical and social implications, constantly motivates an intense research activity in the field of traffic flow modeling. Both applied mathematicians and engineers are involved and several interesting results have been obtained despite the great complexity of the above system, which appears to be rather difficult to be constrained into a mathematical framework. Due to the high complexity of the problems, analytical approaches are infeasible. Current approaches are simulation based (Leveque [3]) which is driven by necessity, but largely enhanced by the widespread availability of computing power nowadays.

In densely populated areas, there is only limited space available for extension of the transport system; and we face increasing pollution and growing accident frequencies as the downsides of mobility. One of the basic goals of studying traffic flow is to understand traffic congestion and look for ways to provide efficient movement of traffic which minimize congestion problems.

Zhang [7] developed a finite difference scheme for a previously reported non-equilibrium traffic flow model. This scheme is an extension of Godunov's scheme (see Leveque [3]) to systems. It utilizes the solutions of a series of Riemann problems at cell boundaries to construct approximate solutions of the non-equilibrium traffic flow model under general initial conditions. Moreover, the Riemann solutions at both left (upstream) and right (downstream) boundaries of a highway allow the specification of correct boundary 
conditions using state variables (e.g., density and/or speed) rather than fluxes. Preliminary numerical results indicate that the finite difference scheme correctly computes entropysatisfying weak solutions of the original model.

In order to investigate efficient numerical scheme for the Lighthill-Whitham-Richards (LWR) traffic flow model, Gani et al. [6] studied a finite difference scheme for the LWR model appended with a linear velocity density relation. This paper performs stability analysis and establishes a physical constraint condition for the implementation of the first order LWR model.

The first order traffic flow model doesn't describe the physical tendency of traffic diffusivity. Therefore, in order the study the effect of traffic diffusivity behavior in the numerical scheme, in [9], authors consider a second order traffic flow model with diffusion term, first introduced by Payne [8]; Kuhne [4] added a diffusive term. The model describes traffic phenomena resulting from interaction of many vehicles by discussing the fundamental traffic variables like density, velocity and flow. In particular, a linear velocity-density relationship which yields a quadratic flux-density relation forms the traffic model as a second order convection-diffusion type partial differential equation. The analytical solution of the second order traffic flow model is developed by using ColeHopf transformation. A study of finite difference scheme with stability analysis is also performed in [9].

In the present work, we develop computer programming code for the implementation of the numerical schemes that has been developed in [9]. Error estimation is produced and numerical feature of rate of convergence is observed by graphical presentation of error profiles for consistently varied discretization parameters. We present numerical simulation results in order to verify some qualitative traffic flow behavior for various traffic parameters.

\section{Fluid Dynamical Models for Traffic Flow}

The fluid-dynamical traffic flow model is used to study traffic flow by collective variables such as traffic flow rate (flux) $q(t, x)$, traffic speed $v(t, x)$ and traffic density $\rho(t, x)$, all of which are funciotns of space, $x \in \mathbf{R}$ and time, $t \in \mathbf{R}^{+}$. The well known first order LWR traffic flow model [2] based on the principle of mass conservation reads as

$$
\frac{\partial}{\partial}+\frac{\partial}{\partial}=0, \quad x \in \mathbf{R}, t>0
$$

Lighthill, Whitham and Richards considered that traffic is an inviscid but compressible fluid (fluid-dynamic model). Densities, speed values and flows were defined as continuous variables in each point in time and space (continuum, macroscopic model). Instead, in 1971, Payne replaced the assumption of instantaneous adaption in the Lighthill-Whitham theory by an equation for inertia, which is similar to a Navier-Stokes equation [8]. Kuhne added a viscosity term in 1984 and initiated using the methods of nonlinear dynamics for analyzing the equation [4], which yields

$$
\frac{\partial}{\partial}+\frac{\partial}{\partial}(q-D \nabla \rho)=0
$$


The term $-D \nabla \rho$ known as Fickian term is introduced to account for external noise, i.e., the resultant of all random fluctuations in road conditions, drivers' response to stimuli, changes in wind, engine power, braking variability, etc. Considering these dissipations the LWR traffic flow model (2.1) can be developed using the equation (2.2), which can be written as

$$
\frac{\partial}{\partial}+\frac{\partial}{\partial}=D \frac{\partial^{2} \rho}{\partial^{2}}
$$

This is a second order PDE and is called the second order traffic flow model.

The second order traffic flow model (2.3) is parabolic while first order LWR traffic flow model (2.1) is hyperbolic. One major difference is that (2.3) always has smooth solutions for $t>0$ even if the initial data $\mu_{0}(x)$ is discontinuous. We can view (2.1) as an approximation to (2.3) valid for $D$ very small, but we may need to consider the effect of $D$ in order to properly interpret discontinuous solution of (2.1)

The solution of (2.3) is again non-dispersive wave with phase and group velocity $q^{\prime}$. The difference is that $D$ introduces dissipation (damping) of the wave. The amplitude decays as $e^{-\nu k^{2}}$, where $k$ is the wave number. This reflects the intuitively reasonable effect that traffic jams should tend to dissolve under homogeneous and stationary conditions.

For a further development $q(\mu)$ has to be specified. Inserting a linear velocity-density closure relationship [2]

$$
v(\rho)=v_{m} \quad\left(1-\frac{\rho}{\rho_{m}}\right)
$$

the flux takes the form

$$
q(\mu)=\rho(\rho)=v_{m} \quad\left(\rho-\frac{\rho^{2}}{\mu_{m}}\right)
$$

Using (2.5) second order traffic flow model (2.3) leads to formulate a linear second order partial differential equation of the form

$$
\frac{\partial}{\partial}+\frac{\partial}{\partial}\left(v_{m}\left(\rho-\frac{\rho^{2}}{\mu_{m}}\right)\right)=D \frac{\partial^{2} \rho}{\partial^{2}}
$$

The linear velocity-density relation (2.4) satisfies the qualitative physical properties

$$
\left.\begin{array}{l}
v(\mu=0)=v_{m} \\
\frac{d}{d} \leq 0 \\
v\left(\rho=\rho_{m} \quad\right)=0
\end{array}\right\}
$$

The corresponding flux-density function (2.5) is parabolic concave up and satisfies the qualitative properties

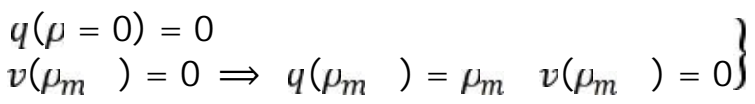


The maximum traffic flow rate under these assumptions occurs at the mid-point of the fundamental diagram, for $\rho=\frac{\mu_{m}}{2}$, and the maximum flow is

$$
\varphi_{m}=\frac{1}{4} \rho_{m} \quad v_{m}
$$

\section{Analytical Solution of the Second Order Traffic Flow Model}

The second order traffic flow model (2.6) can be linearly mapped onto convection diffusion equation (CDE) under certain conditions. Then obtained equation can be solved if we know the traffic density at a given initial time, i.e., if we know the traffic density at a given initial time $t_{0}=0$ we can predict the traffic density for all future time $t>t_{0}$, in principle. In [9] authors present the analytic solution of the second order traffic flow model as an initial value problem (IVP) in the form

$\rho(t, x)=D \frac{\rho_{m}}{v_{m}} \frac{\partial}{\partial}\left(\ln \left\lfloor\frac{1}{\sqrt{4 \pi}} \int_{-\infty}^{\infty} e \quad\left(-\frac{\left(v_{m} t-x-z\right)^{2}}{4 D}-\frac{v_{m}}{D_{m}} \int_{0}^{z} \rho_{0}\left(x^{\prime}\right) d x^{\prime}\right) d\right\rfloor\right)$

where $\rho_{\mathrm{u}}(x)=\rho(0, x)$ is the initial condition.

However, in reality it is very complicated to approximate the initial density $\rho_{0}(x)$ of the Cauchy problem as a function of $x$ from given initial data, e.g. it may cause a huge error. Moreover, the analytical solution given by equation (3.1) depends on the initial value function under a complex double integral and it is quite difficult to evaluate the value of the double integral of the solution.

Therefore, there is demand of efficient numerical methods for solving the second order traffic flow model.

\section{Finite Difference Method for Second Order Traffic Flow Model}

As we have already seen in the previous section that the analytic solution of the second order traffic flow problem is in a complex form, one need to employ numerical method for the model.

We consider our second order traffic flow model with two sided boundary condition as an initial boundary value problem (IBVP):

$$
\begin{aligned}
& \text { with I.C. } \left.\rho\left(t_{\mathrm{U}}, x\right)=\rho_{\mathrm{U}}(x) ; a \leq x \leq b\right\} \\
& \text { and B.C. } \rho(t, a)=\mu_{u}(t) ; t_{0} \leq t \leq T \\
& \left.\rho(t, b)=\rho_{b}(t) ; t_{0} \leq t \leq T\right) \\
& \text { where } \quad q(\rho)=\rho=\mu v_{m}\left(1-\frac{\rho}{\mu_{m}}\right)
\end{aligned}
$$

\subsection{Explicit Backward-Centered (FTBSCS) Difference Scheme}

We study finite difference method for the parabolic type PDE [3]. For our model as IBVP we discretize $\frac{\partial}{\partial}$ by the first order forward difference formula in time, $\frac{\partial}{\partial}$ by the first 
order backward difference formula in space and $\frac{\partial^{2} p}{\partial^{2}}$ by the second order central difference formula in space at any point $\left(t^{n}, x_{i}\right)$ for $i=1, \ldots \ldots, M-1 ; n=$ $0,1, \ldots \ldots, N-1$, considering $\rho\left(t^{n}, x_{i}\right)=\mu_{l}^{n}$, we have

$$
\begin{aligned}
& \frac{\partial \rho_{i}^{n}}{\partial} \approx \frac{\rho_{i}^{n+1}-\rho_{i}^{n}}{\Delta t} \\
& \frac{\partial\left(\rho_{i}^{n}\right)}{\partial} \approx \frac{q\left(\rho_{i}^{n}\right)-q\left(\rho_{i-1}^{n}\right)}{\Delta x} \\
& \frac{\partial^{2} \rho_{i}^{n}}{\partial^{2}} \approx \frac{\rho_{i+1}^{n}-2 \rho_{i}^{n}+\rho_{i-1}^{n}}{\Delta x^{2}}
\end{aligned}
$$

Using (4.3), (4.4) and (4.5) the numerical discretization of (4.1) reads as

$$
\rho_{i}^{n+1}=\left(1-2 \frac{D \Delta t}{\Delta x^{2}}\right) \rho_{i}^{n}+\frac{D \Delta t}{\Delta x^{2}}\left(\rho_{i-1}^{n}+\rho_{i+1}^{n}\right)-\frac{\Delta t}{\Delta x}\left(q\left(\rho_{i}^{n}\right)-q\left(\rho_{i-1}^{n}\right)\right)
$$

which is known as FTBSCS difference scheme for the IBVP (4.1).

However the scheme is not straight forward to implement. To ensure the convergence of the scheme, one needs to satisfy stability condition for the scheme. For our traffic flow model we perform stability conditions and determine the stability conditions as follows.

\subsection{Explicit Centered-Centered (FTCSCS) Difference Scheme}

For the FTCSCS Difference scheme we discretize $\frac{\partial}{\partial}$ by the first order forward

difference in time, $\frac{\partial}{\partial}$ by the first order central difference in space and $\frac{\partial^{2} \mu}{\partial{ }^{2}}$ by the second order central difference in space at any point $\left(t^{n}, x_{i}\right)$ for $i=1, \ldots \ldots, M-1 ; n=$ $0, \ldots \ldots, N-1$.

$$
\begin{aligned}
& \frac{\partial}{\partial} \approx \frac{\mu_{i}^{n+1}-\mu_{i}^{n}}{\Delta \mathrm{t}} \\
& \frac{\partial}{\partial} \approx \frac{q_{i+1}^{n}-q_{i-1}^{n}}{2 \Delta x} \\
& \frac{\partial^{2} \rho}{\partial^{2}} \approx \frac{\rho_{i+1}^{n}-2 \mu_{i}^{n}+\mu_{i-1}^{n}}{\Delta x^{2}}
\end{aligned}
$$

Using (4.7), (4.8) and (4.9) the discretization of (4.1) is

$$
\rho_{i}^{n+1}=\left(1-2 \frac{D \Delta t}{\Delta x^{2}}\right) \mu_{i}^{n}+\frac{D \Delta t}{\Delta x^{2}}\left(\rho_{i+1}^{n}+\mu_{i-1}^{n}\right)-\frac{\Delta t}{2 \Delta x}\left(\varphi_{i+1}^{n}-q_{i-1}^{n}\right)
$$

which is known as FTCSCS Difference scheme for IBVP (4.1).

The stability condition and physical constraints condition for the above two schemes is established in [9] as follows: 
Lemma 4.1: Stability of the FTBSCS difference scheme (4.6) is given by the conditions

$$
0 \leq \frac{q^{\prime}\left(\rho_{i}^{n}\right) \Delta t}{\Delta x} \leq 1 \quad \text { and } \quad 0 \leq \frac{D \Delta t}{\Delta x^{2}} \leq \frac{1}{2}
$$

Lemma 4.2: Physical constraints condition and the stability condition $0 \leq \frac{q^{\prime}\left(p_{l}^{n}\right) \Delta t}{\Delta x} \leq 1$ are assured by the simultaneous conditions

$$
\frac{v_{m} \Delta t}{\Delta x} \leq 1 \text { and } \rho_{m}=\alpha \max _{i} \rho_{i}^{\mathrm{U}} ; \alpha \geq 2
$$

Theorem 4.1: The stability of the FTBSCS difference scheme (4.6) and physical constraint condition $q^{\prime}(\mu) \geq 0$ can be guaranteed by the simultaneous conditions

$$
\frac{v_{m} \Delta t}{\Delta x} \leq 1 \text { and } \rho_{m}=\alpha \max _{i} \rho_{i}^{0} ; D=\frac{\beta}{2} v_{m} \quad \Delta x, \alpha \geq 2, \beta \geq 1
$$

\section{Numerical Simulation}

In this section we implement the finite difference scheme of the second order traffic flow model by computer programming and perform numerical simulation for some specific cases.

For the spatial domain $\left[\begin{array}{ll}0 & 10\end{array}\right]$ in kilometer we choose the maximum velocity of cars is $v_{m}=60 \mathrm{~km} /$ hours and the maximum density $\mu_{m}=500 \mathrm{cars} / \mathrm{Km}$. We perform the numerical experiment for 3 minutes in 361 time steps (with temporal grid sizes $0.5 \mathrm{sec}$ ) and 201 spatial grid points (with spatial grid size $.05 \mathrm{~km}$ ) for the highway.

Now we consider the initial density as shown in Figure 5.1 at $t=0$ and boundary condition is obtain by the analytical solution describe in section 3. Figure 5.1 shows the propagating traffic waves at first minutes, second minutes and third minutes for value of diffusion constant $\mathrm{D}=0.1 \mathrm{Km}^{2} / \mathrm{Min}$.
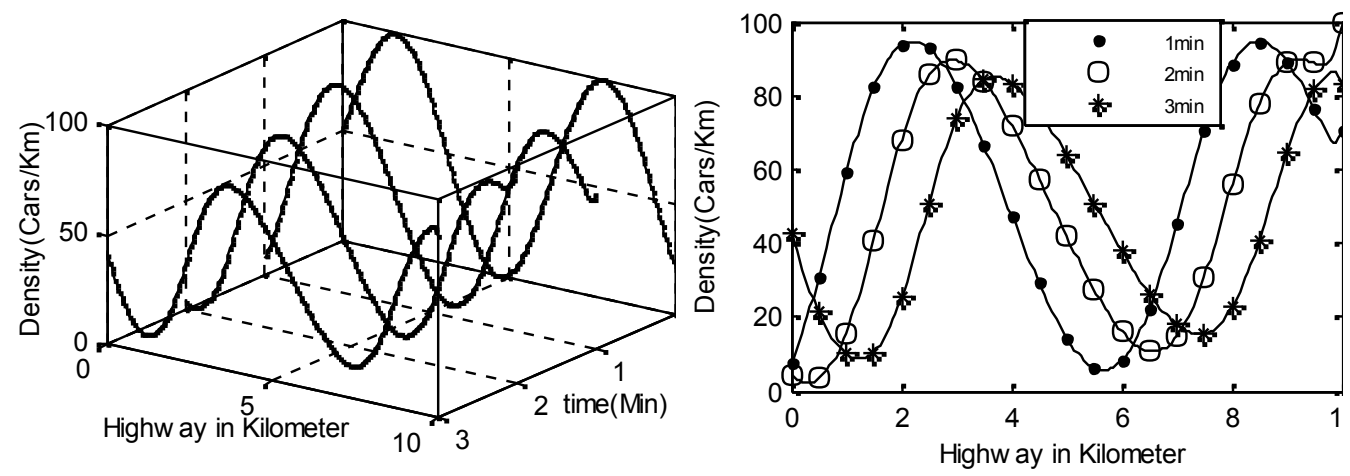

Fig. 5.1 Density Profile (Explicit backward-centered (FTCSCS) difference scheme) 
Figure 5.2 and 5.3 shows the corresponding velocity $v(t, x)$ and flux $q(t, x)$ profiles.
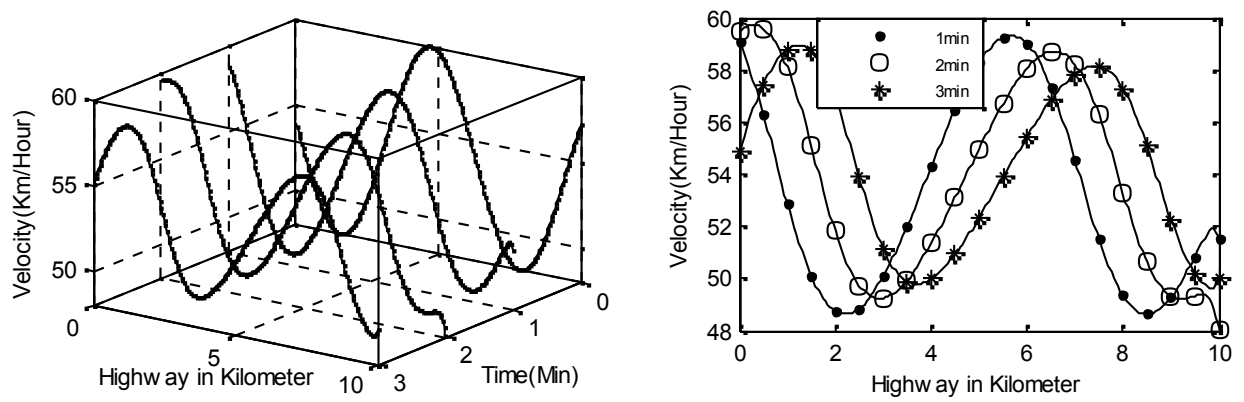

Fig. 5.2 Velocity Profile(Explicit backward-centered (FTCSCS) difference scheme)
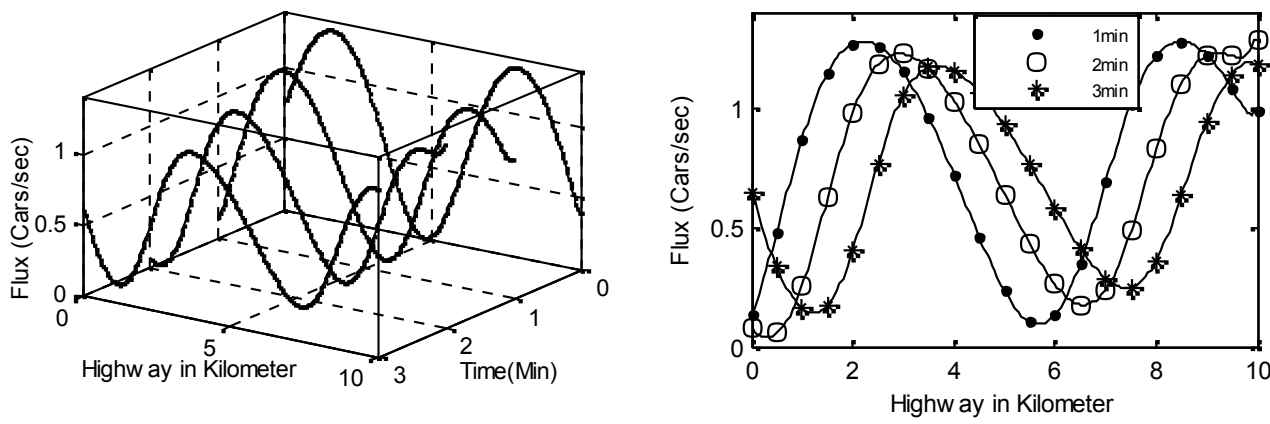

Fig. 5.3 Flux Profile (Explicit backward-centered (FTCSCS) difference scheme)

For the different diffusion constants the effects of propagating traffic density is shows in figure 5.4(a) and 5.4(b) at time 3 minutes and 6 minutes respectively.

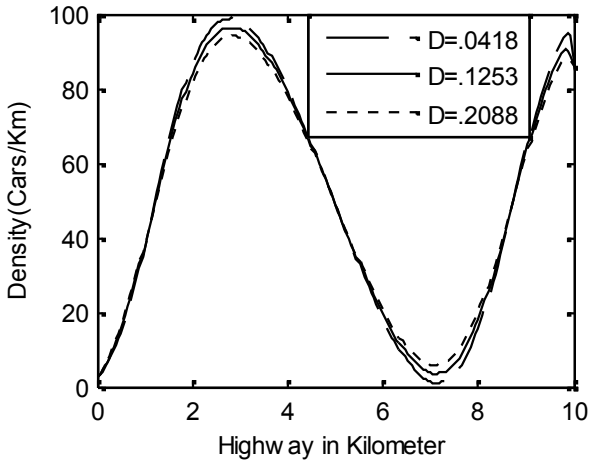

Fig. 5.4(a): Density at $t=3$ minitues

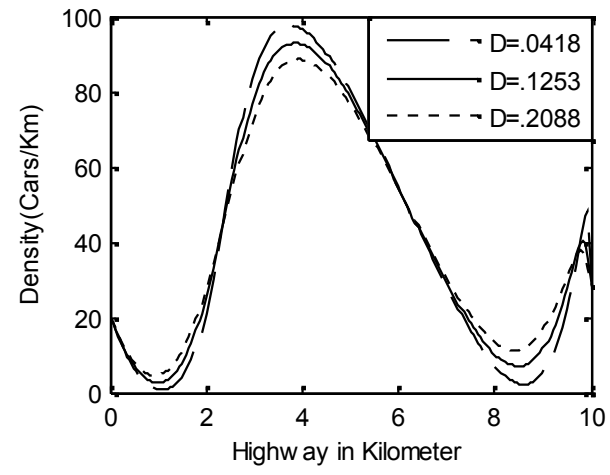

Fig. 5.4(b): Density at $t=6$ minitues

\section{Error Estimation}

To estimate the error of our numerical schemes, we choose diffusivity constant $\mathrm{D}=$ $0 \mathrm{~km}^{2} / \mathrm{min}$. Then the traffic flow model that we considered becomes 


$$
\frac{\partial}{\partial}+\frac{\partial}{\partial}\left(v_{m}\left(\mu-\frac{\rho^{2}}{\mu_{m}}\right)\right)=0
$$

Using method of characteristics the analytical solution of this model with the initial condition $\rho\left(t_{\mathrm{U}}, x\right)=\rho_{\mathrm{U}}(x)$ is presented in [6] as

$$
\mu(t, x)=\mu\left(0, x_{0}\right)=\mu_{0}\left(x_{0}\right)=\mu_{0}\left(x-v_{m}\left(1-\frac{2 \mu}{\mu_{m}}\right) t\right)
$$

To evaluate the accuracy of our numerical schemes we consider the initial condition $\mu_{0}(x)=\frac{1}{2} x$ then we have

$$
\begin{aligned}
\rho(t, x) & =\frac{1}{2}\left(x-v_{m} \quad\left(1-\frac{2 \rho}{\mu_{m}}\right) t\right) \\
\Rightarrow \rho(t, x) & =\frac{\rho_{m}\left(x-v_{m} t\right)}{2\left(\mu_{m}-v_{m} t\right)}
\end{aligned}
$$

We prescribe the corresponding two-sided boundary values by the equations

$$
\rho_{u}(t)=\frac{\rho_{m}\left(x_{u}-v_{m} t\right)}{2\left(\rho_{m}-v_{m} t\right)}
$$

and $\quad \rho_{b}(t)=\frac{\rho_{m}\left(x_{b}-v_{m} t\right)}{2\left(\rho_{m}-v_{m} t\right)}$

For the above initial and boundary conditions with maximum velocity $v_{m}=$ $60 \mathrm{~km} /$ hour and maximum density $\mu_{m}=500 \mathrm{cars} / \mathrm{km}$ in the spatial domain $[5,15]$ in kilometer with 101 spatial grids points with step size $x=0.1 \mathrm{~km}=100$ meter, we perform the numerical experiment of a highway of $10 \mathrm{~km}$ for 3 minutes. We compute the relative error in $L_{1}$-norm defined by:

$$
\|e\|_{1}=\frac{\left\|\rho_{e}-\rho_{h}\right\|_{1}}{\left\|\rho_{h}\right\|_{1}}
$$

for all time where $\rho_{e}$ is the exact solution and $\rho_{h}$ is the numerical solution computed by our numerical schemes.

Figure 5.5 and 5.6 shows the relative errors for explicit FTBSCS difference scheme and the relative error for explicit FTCSCS difference scheme respectively. In both cases the relative error remains below $8 \times 10^{-8}$ which is quite acceptable. It is obseved that the error is decreasing with respect to the smaller descretization parameters $t$ and $x$, which shows a very good feature of convergence of our schemes. 


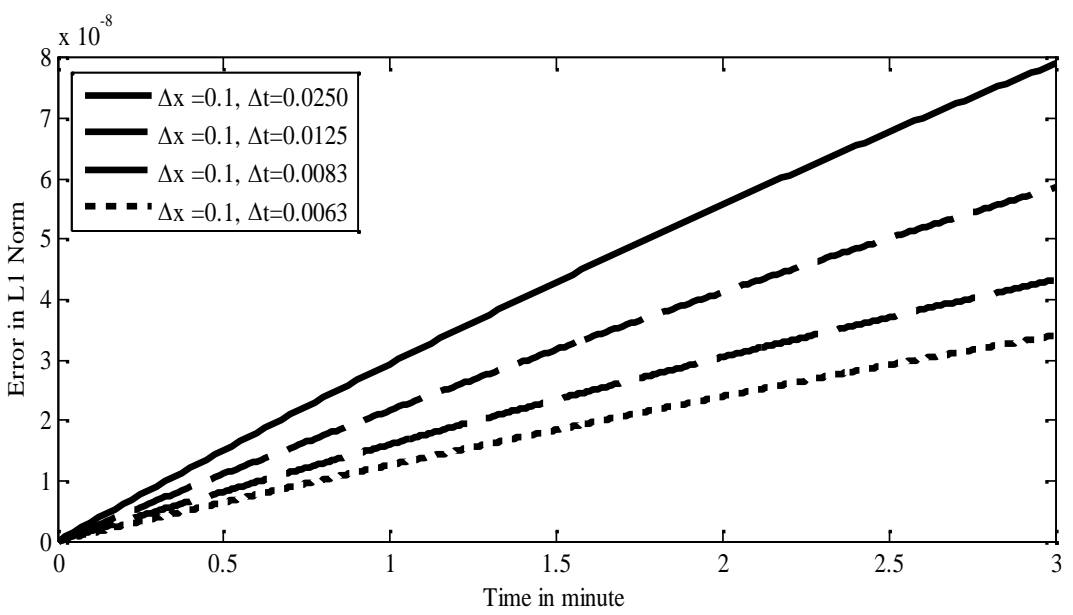

Fig. 5.5 Convergence of explicit FTBSCS difference scheme

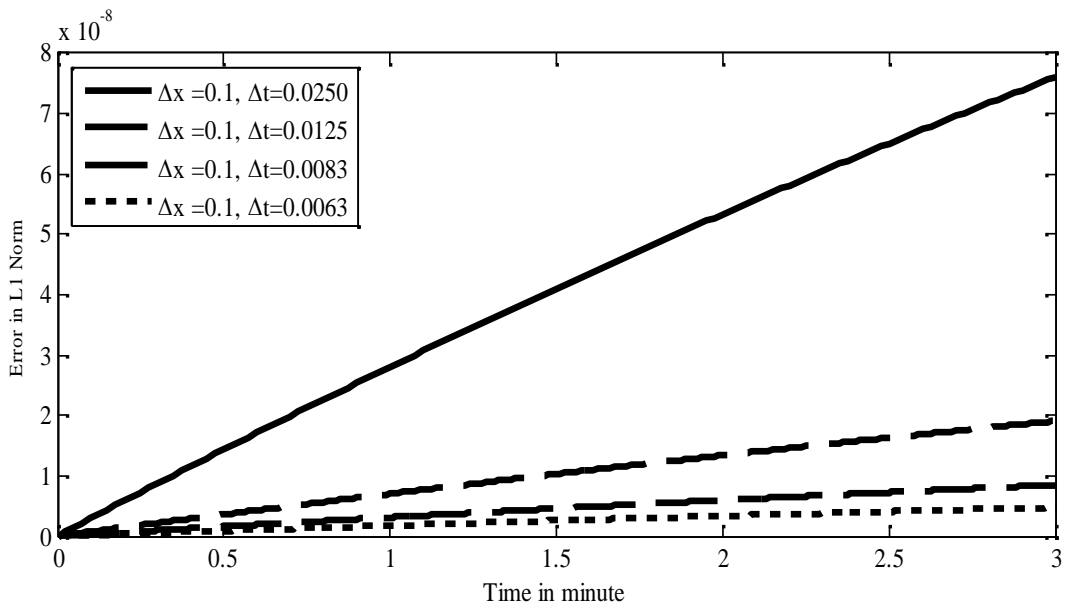

Fig. 5.6 Convergence of explicit FTCSCS difference scheme

Though both schemes shows a very good feature of convergence, the relative error of explicit FTCSCS difference scheme decrease more quickly than explicit FTBSCS difference scheme for smaller time step $t$. Hence the accuracy of explicit FTCSCS difference scheme is better than explicit FTBSCS difference scheme.

\section{Conclusion}

In this work, analytic solution for simplified initial condition is used for error estimation of numerical method for the model and the rate of convergence is observed by graphical presentation of error profiles for consistently varied discretization parameters. These features are found quite satisfactory for performing traffic flow simulation. Therefore, we perform numerical computation of the second order model by the finite difference scheme and the computed density, velocity and flux profiles for various flow parameters are 
observed in a good agreement with well known qualitative behavior of the traffic flow. This work can be extended for the traffic flow model with inflow-outflow effect.

\section{REFERENCES}

[1] Klar, A., Kuhne, R. and Wegener, R., Mathematical Models for Vehicular Traffic, Surv. Math. Ind., 6, 215-239, 1996.

[2] Richard Haberman, Mathematical models, Prentice-Hall, 1977.

[3] Randall, J. Leveque, Numerical Methods for Conservation Law, Lectures in Mathematics, ETH-Zurich, Birkhauser-Verlag, Basel, Second Edition, (1992).

[4] R. Kuhne, Macroscopic freeway model for dense traffic stop-start waves and incident detection, $9^{\text {th }}$ Int. symp. Transpn. Traffic Theory, VNU Science Press, 1984.

[5] E. Hopf, The partial differential equation $u_{\tau}+u u_{x}=u_{x}$, Pure Appl. Math. 3, (1950), 201,

[6] M. O. Gani, M. M. Hossain and L. S. Andallah, A Finite Difference Scheme for a Fluid Dynamic Traffic Flow Model Appended with Two Point Boundary Condition, GANIT J. Bangladesh Math. Soc. 31 (2011) 43-52

[7] Zhang, H. M., A finite difference approximation of a non-equilibrium traffic flow model, Transportation Research Part B: Methodologicial Vol. 35, Issue 4 (2001), 337-365.

[8] H.J. Payne, Models of freeway traffic and control, Mathematical Models of Public Systems, Vol.1, Simulation Council, La Jolla, CA, (1971), p. 51.

[9] Tauhedul Azam, S. Ali and L. S. Andallah, An Analytical Method and A Numerical Scheme for the Solution of Second Order Traffic Flow Model, J. J. Math. and Math. Sci. 26(2011), 71-80. 